\title{
Neoplasia intraepitelial de cuello uterino en un área de salud de la Isla de la Juventud, Cuba: 1999-2008
}

\author{
Heenry Dávila Gómez ${ }^{1}$, Abel García Valdés ${ }^{1}$, Félix Álvarez Castillo ${ }^{1}$, Yunior Castillo \\ Blanco 1, Laritza Imengana Fonte ${ }^{1}$, Zaskia Matos Rodríguez. a \\ 1 Hospital General Docente "Héroes del Baire", Isla de la Juventud, Cuba.
}

a Alumno, Escuela de Medicina, Facultad de Ciencias Médicas, Isla de la Juventud, Cuba.

\section{RESUMEN}

Antecedentes: Las neoplasias intraepiteliales cervicales (NIE) constituyen lesiones precursoras del cáncer de cuello uterino, mostrando un incremento de sus tasas de incidencia en todo el mundo. Cuba, a pesar de contar con un programa de tamizaje hace más de 40 años no ha logrado disminuir la mortalidad por este cáncer. Objetivo: Estudio de casos y controles entre los años 1999 y 2008 para determinar los factores de riesgo asociados a la probabilidad de tener una neoplasia intraepitelial cervical en las pacientes del área de salud del Policlínico Universitario "Dr. Juan Manuel Páez Inchausti", Isla de la Juventud, Cuba. Método: La muestra estuvo compuesta por 632 pacientes, de ellos 316 casos con NIE y pareados por edad, a partir de la revisión de sus tarjetas individuales de citología. Resultados: El promedio de edad fue de 36,08 \pm 7,23 años. La mayor diferencia entre los grupos de estudios se encontró entre las pacientes de piel negra. En las pacientes que iniciaron sus relaciones sexuales antes de los 15 años el riesgo de padecer una NIE fue 3,8 veces mayor, triplicándose también el riesgo en las pacientes multíparas y en aquellas que usaron anticonceptivos orales por más de 10 años. Conclusión: Los factores de riesgo más relevantes en la muestra de estudio fueron el inicio precoz de las relaciones sexuales, las infecciones de transmisión sexual y la multiparidad.

\section{PALABRAS CLAVE: Neoplasia intraepitelial, cáncer cervico-uterino, factor de riesgo, virus del papiloma humano}

\section{SUMMARY}

Background: The cervical intraepithelial neoplasm (CIN) constitutes pre-cancer lesions of uterine cervix, showing an increment of their rates of incidence in everybody. Cuba, in spite of having a screening program, it does more than 40 years have not been able to diminish the mortality for this cancer. Objective: A case control study during the period of 1999-2008 was made to determinate the risk' factors associated with the probability of having a CIN in patients of the University Policlinic "Dr. Juan Manuel Paez Inchausti" in the Island of the Youth (Cuba). Method: This study includes 632 patients, the half of them with this illness and the same number of healthy persons. The information about the patients was obtained by revision of his clinical history documents. Result: The age average was from $36.08 \pm 7.23$ years. The more differentiates between both studies groups were between the patients of black skin. In the patients that began their sexual relationships before the 15 years the risk of suffering a CIN was 3.8 times, also tripling the risk in the multipary patient and in those that used oral contraceptive for more than 10 years. Conclusions: The risk 
factors of more relevance were the precocious beginning of the sexual relationships, the infections of sexual transmission and multiparity.

\section{KEY WORD: Intraepithelial neoplasia, cervical cancer, risk factor, human papillomavirus}

\section{INTRODUCCIÓN}

Las neoplasias intraepiteliales cervicales (NIC), también llamadas lesiones escamosas intraepiteliales (LEI) o displasias cervicales, son consideradas como lesiones precursoras del cáncer de cuello uterino, las que han sido objeto de muchos estudios e investigaciones desde hace más de 70 años $(1,2)$. Menos del $5 \%$ de las citologías de cuello uterino resultan en una displasia cervical, trastornos que son más frecuentes en las edades reproductivas, en particular entre 25 a 35 años (3).

Las NIC constituyen un eslabón anterior al desarrollo potencial de un cancer invasor, de ahí, la importancia que tiene la pesquisa para descubrirlas y tratarlas si queremos disminuir la incidencia del cáncer cervical y sus consecuencias para la calidad y expectativa de vida de la mujer.

El cáncer del cérvix es la lesión maligna del aparato genital femenino más frecuente en América Latina y el Caribe (4). Se calcula que cada año se presentan alrededor de 68.000 casos nuevos de este cáncer, sólo en la región de las Américas, la gran mayoría diagnosticados durante la etapa reproductiva (1).

El cáncer de cuello uterino fue la primera causa de mortalidad por cáncer entre las mujeres colombianas en el 2002, y durante el periodo 1995-1999 fue la primera causa de muerte relacionada con la salud sexual y reproductiva de la mujer. A pesar de que Colombia alcanzó una cobertura de citología superior al $70 \%$ tras más de quince años de introducir los programas de tamización, no ha logrado disminuir significativamente la mortalidad por esta causa. Un estudio en ese país, González y cols (5), analizaron 4.957 citologías, encontraron un 39,9\% de exámenes anormales, con $4,3 \%$ de lesiones intraepiteliales de bajo grado (NIC I), $1,2 \%$ de lesiones de alto grado (NIC II, NIC III, carcinoma in situ o CIS) y $0,1 \%$ de carcinoma infiltrante. De las 762 pacientes con citología alterada en el $48,3 \%$ se confirmó por biopsia algún cambio neoplásico. En Estados Unidos de Norteamérica durante el 2003 se diagnosticaron aproximadamente $600.000 \mathrm{ca}-$ sos de cáncer cervical (6).

Cuba cuenta con un Programa de Pesquisaje desde el año 1968, en el que millones de mujeres han sido sometidas al estudio de la citología cérvico vaginal y miles de ellas han sido beneficiadas con el diagnóstico temprano de esta enfermedad. En el periodo comprendido entre 1994 y el 2000 fueron diagnosticadas a través del Programa Nacional de Diagnóstico Precoz del Cáncer Cérvico Uterino 20.817 neoplasias intraepiteliales cervicales, que se distribuyeron en $8.488 \mathrm{NIC} \mathrm{I}(40,8 \%), 7.527 \mathrm{NIC}$ II $(36,1 \%)$ y $4.802 \mathrm{NIC} \mathrm{III}(23,1 \%)(7)$. En el mismo periodo, un promedio del $15 \%$ de los cánceres de cuello uterino diagnosticados, fue en estadio clínico microinvasor o infiltrante (8). En el 2006 hubo un total de 473 defunciones con una tasa de 8,4 por cada 100.000 mujeres (9).

Se han descrito varios factores asociados a la aparición de patologías cervicales tales como: inicio precoz de las relaciones sexuales (10), multiparidad, múltiples parejas sexuales y conducta sexual riesgosa (tanto de la mujer como de su pareja sexual), el humo del cigarro, las infecciones de transmisión sexual, el déficit vitamínico y factores hormonales, no obstante existen datos que confirman el papel relevante de las infecciones por el papiloma virus humano (HPV), así como la inmunodeficiencia exógena o endógena en el desarrollo de las displasias cervicales $(11,12)$. La relación de la infección genital por algunos tipos de virus del papiloma humano (VPH) en la patogenia de las NIC y del cáncer cérvico uterino es reconocida hace más de 20 años en muchas publicaciones $(13,14)$.

Existen varios factores que dificultan el diagnóstico de la neoplasia del cuello uterino: la clínica es generalmente nula, las infecciones son frecuentes, la paciente tiene temor de acudir al médico ya que piensa que el chequeo periódico no es necesario, además priorizan otras funciones en el hogar y el trabajo (15).

Hay quienes piensan que los casos de NIC progresan por estos estados antes de desarrollar un cáncer (16). Sin embargo, si bien es cierto que los cánceres cérvico-uterinos, en especial los invasores de células escamosas, vienen precedidos por una fase de enfermedades pre-invasoras de largo tiempo, existen evidencias que el cáncer de cuello puede ocurrir sin que haya una detectable progresión a lo largo de los tres estadios y que una neoplasia intraepitelial de alto grado puede ocurrir sin que primero haya existido como una lesión de bajo grado (3). 
Más del $80 \%$ de las lesiones epiteliales de bajo grado (LEBG) y el $100 \%$ de las lesiones epiteliales de alto grado (LEAG) están asociados con VPH de alto riesgo. VPH 16 es el tipo de virus del papiloma humano asociado con mayor frecuencia en ambos tipos de lesiones. Aunque la mayoría de las LEAG derivan de LEBG, aproximadamente el $20 \%$ de los casos de LEAG aparecen "de novo", sin LEBG previos. Las tasas de progresión no son uniformes y aunque sobre todo el VPH 16 está asociado con un riesgo elevado, es difícil predecir la evolución en una persona en particular. El riesgo de desarrollar cáncer depende sólo en parte del subtipo de VPH, ya que también depende del estado inmunitario de la persona y de condiciones ambientales. La progresión hacia carcinoma invasivo, si llega a ocurrir, puede ocurrir en pocos meses o producirse durante más de una década (17).

La Isla de la Juventud, sigue siendo el territorio de Cuba con mayor porcentaje de casos de neoplasias malignas en comparación con la población total. Durante el año 2006 el territorio reportó una tasa de mortalidad ajustada por cáncer para todas las edades de 141,3 fallecidos por 100.000 habitantes, tasa 1,2 veces mayor a la del país $(117,6$ fallecidos por 100.000 habitantes) (18). Por ser esta área de salud, la de mayor incidencia de casos, nos motivamos a realizar este estudio en la búsqueda de los principales factores de riesgo que están influyendo en tal comportamiento.

\section{MÉTODO}

Se realizó un estudio de casos y controles para determinar los factores de riesgo asociados a la probabilidad de tener una neoplasia intraepitelial (NIE) en las pacientes del área de salud del Policlínico Universitario "Dr. Juan Manuel Páez Inchausti", durante el periodo comprendido entre los años 1999 y 2008. Se estudiaron 316 casos e igual número de controles, escogidos por muestreo aleatorio simple según edad y lugar de procedencia (urbano o rural). Se estudió la edad de las pacientes, el color de piel, la menarquía, la edad de inicio de las relaciones sexuales, la presencia de infecciones cérvico-vaginales y/o HPV (por citología/biopsia), la paridad y la edad en la que se produjo el primer parto, el uso de anticonceptivos hormonales y el hábito tabáquico. Los datos fueron obtenidos de sus historias clínicas y procesados con Epilnfo 6.04d, determinando medidas de resumen para las variables estudiadas. La estimación de riesgo se hizo en función del valor de Odds Ratio (OR) y una probabilidad $p<0,05$ como grado de significación estadística.

\section{RESULTADOS}

En la muestra de estudio el promedio de edad fue de 36,08 $\pm 7,23$ años. El análisis de la prevalencia de NIE según rangos de edad mostró que en el rango de 35-39 años se observó la mayor proporción de casos con relación al universo de cada grupo etáreo $(8,3 \%)$ lo que significó un riesgo de 2,6 veces mayor con relación a los demás grupos, seguido del rango de 30-34 años con 7,8\% y un riesgo 2,3 veces mayor. En los rangos de 25-29 años $(O R=1,3)$ y 40-44 años $(O R=1,1)$ también se encontró una diferencia estadística significativa, aunque no constituyeron por su valor rangos con significación de riesgo. Los grupos de 50-54 años (OR=0,12), 55-59 años $(\mathrm{OR}=0,08)$ y de 60 años o más $(\mathrm{OR}=0,02)$ representaron los rangos de menor riesgo (Tabla I).

En cuanto al color de piel, la mayor diferencia entre los grupos de estudios se encontró entre las pacientes de piel negra $(12,3 \%)$ y $(5,1 \%)$ en los controles, riesgo 2,6 veces mayor al resto de las pacientes. En las pacientes de piel blanca o mestiza no se encontró diferencias significativas (Figura 1).

En nuestra muestra podemos ver como las mujeres que tuvieron su menarquia después de los 16 años mostraron una diferencia significativa, pues casi duplicaron el porcentaje de controles, con un OR cercano a los valores de riesgo, seguidas en orden de relevancia por aquellas que tuvieron su menarquia antes de los 12 años, donde la diferencia entre casos y controles también fue significativa. En aquellas mujeres que tuvieron su menarquia entre los 12 y 15 años, el $O R$ mostró un efecto protector $(O R<1)$.

Otro elemento de interés relacionado con el comienzo de la capacidad reproductiva es la edad con que se inician las relaciones sexuales en la mujer, la cual tuvo significación estadística cuando estas comenzaron antes de los 15 años, encontrando un $13,9 \%$ en los casos por un $4,1 \%$ en los controles, lo que representó un riesgo 3,8 veces mayor. También se encontró significación de riesgo entre las pacientes que iniciaron sus relaciones sexuales entre $15-16$ años, al observar un $36,4 \%$ en los casos por un $16,1 \%$ en los controles, representando un riesgo tres veces mayor (Figura 2).

Específicamente dentro de las infecciones cérvico vaginales, denota gran importancia etiológica la infección por el virus del papiloma humano (HPV), identificado por todos como el principal agente causal. En la Tabla II podemos ver como también fue significativa la diferencia entre casos y controles en cuanto a la infección por este germen, pues mientras estuvo presente en 1 de cada 8 casos solo se encontró en 1 de cada 25 controles $(\mathrm{OR}=3,5)$. 
Tabla I

\section{DISTRIBUCIÓN DE PACIENTES CON NEOPLASIA INTRAEPITELIAL (NIE) DE CUELLO UTERINO SEGÚN GRUPOS ETÁREOS}

\begin{tabular}{ccccccc}
\hline Edad (años) & NIE & Universo & $\%$ & OR & IC 95\% & Valor $p$ \\
\hline$<25$ & 5 & 146 & 3,42 & 0,83 & $0,30-2,11$ & 0,6795 \\
$25-29$ & 54 & 1.064 & 5,08 & 1,30 & $0,95-1,78$ & 0,0826 \\
$30-34$ & 80 & 1.032 & 7,75 & 2,30 & $1,75-3,01$ & 0,0000 \\
$35-39$ & 89 & 1.075 & 8,28 & 2,55 & $1,96-3,31$ & 0,0000 \\
$40-44$ & 47 & 1.035 & 4,54 & 1,13 & $0,81-1,57$ & 0,4375 \\
$45-49$ & 30 & 1.084 & 2,77 & 0,63 & $0,42-0,94$ & 0,0173 \\
$50-54$ & 6 & 1.046 & 0,57 & 0,12 & $0,05-0,28$ & 0,0000 \\
$55-59$ & 4 & 1.063 & 0,38 & 0,08 & $0,02-0,21$ & 0,0000 \\
$\geq 60$ & 1 & 1.038 & 0,10 & 0,02 & $0,00-0,13$ & 0,0000 \\
Total & 316 & 7.399 & 4,27 & & &
\end{tabular}

Fuente: Registro de Citologías Orgánicas. Policlínico Universitario “Dr. Juan Manuel Páez Inchausti”.

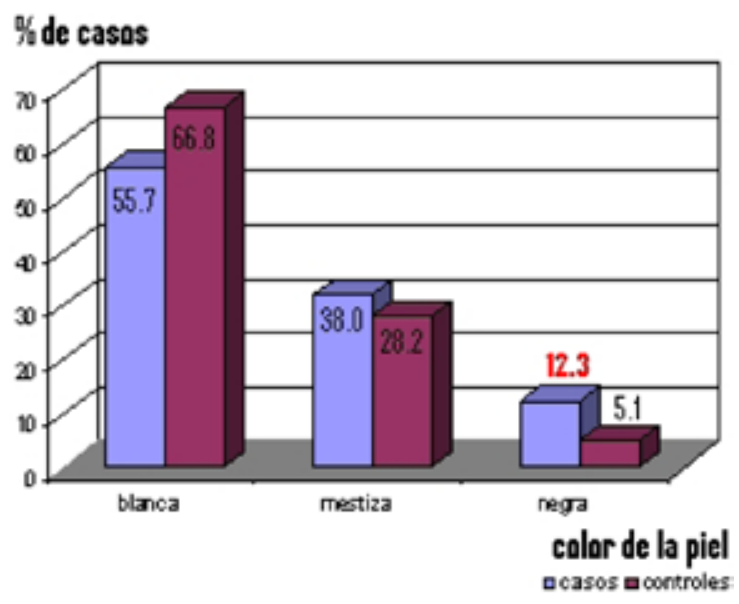

Figura 1. Distribución de pacientes con neoplasia intraepitelial de cuello uterino según color de la piel.

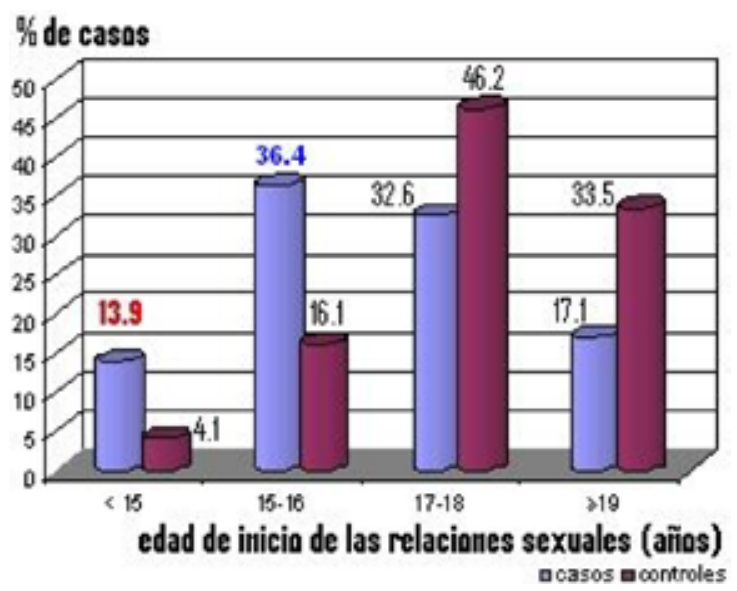

Figura 2. Distribución de pacientes con neoplasia intraepitelial de cuello uterino según edad de inicio de las relaciones sexuales.

Tabla II

\section{DISTRIBUCIÓN DE PACIENTES CON NEOPLASIA INTRAEPITELIAL (NIE) DE CUELLO UTERINO SEGÚN PRESENCIA DE INFECCIÓN POR EL VIRUS DEL PAPILOMA HUMANO (HPV)}

\begin{tabular}{lcccccrr}
\hline Infección por HPV & \multicolumn{2}{c}{ Casos } & \multicolumn{2}{c}{ Controles } & OR & IC 95\% & Valor $p$ \\
& NIE & $\%$ & NIE & $\%$ & & & \\
\hline SI & 39 & 12,34 & 12 & 3,80 & 3,57 & $1,76-7,36$ & 0,0001 \\
NO & 277 & 87,66 & 304 & 96,20 & 0,28 & $0,14-0,57$ & 0,0001 \\
Total & 316 & 100,00 & 316 & 100,00 & & & \\
\hline
\end{tabular}


Hubo diferencia significativa de presentar NIE entre aquellas mujeres que tuvieron 4 o más partos $(\mathrm{OR}=3,4)$ (Figura 3). El primer parto antes de los 17 años fue un factor de riesgo significativo de presentar NIE $(\mathrm{OR}=2,12)$ (Tabla III).

El consumo de anticonceptivos por más de 10 años fue un factor de riesgo significativo de presentar NIE $(\mathrm{OR}=3,3)$ (Tabla IV); no hubo diferencia significativa en rangos de tiempo de menor consumo.

\section{DISCUSIÓN}

En el estudio que se presenta se encontró que la neoplasia intraepitelial fue más frecuente entre los 30-39 años de edad, en los pacientes de piel negra, en aquellas que iniciaron sus relaciones sexuales antes de los 17 años de edad, multíparas, en aquellas que tuvieron su primer parto antes de los 17 años de edad y en aquellas que usaron anticonceptivos hormonales por más de 10 años.

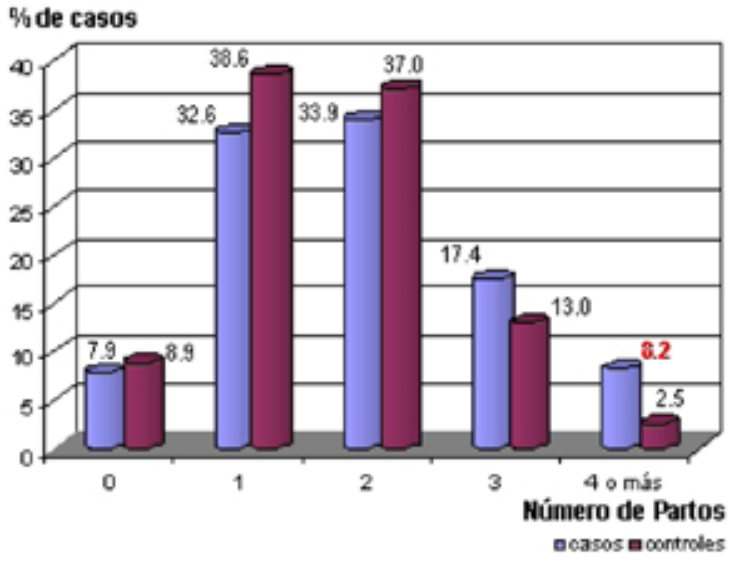

Figura 3. Distribución de pacientes con neoplasia intraepitelial de cuello uterino según número de partos.

Tabla III

\section{DISTRIBUCIÓN DE PACIENTES CON NEOPLASIA INTRAEPITELIAL (NIE) DE CUELLO UTERINO SEGÚN EDAD DEL PRIMER PARTO}

\begin{tabular}{lccccccr}
\hline Edad 1er parto & \multicolumn{2}{c}{ Casos } & \multicolumn{2}{c}{ Controles } & OR & IC $95 \%$ & Valor $p$ \\
& NIE & $\%$ & NIE & $\%$ & & & \\
\hline$<17$ & 59 & 20,14 & 28 & 9,66 & 2,12 & $1,33-3,39$ & 0,0008 \\
$17-19$ & 68 & 23,21 & 85 & 29,31 & 0,78 & $0,54-1,13$ & 0,1780 \\
$20-24$ & 112 & 38,23 & 91 & 31,38 & 1,27 & $0,90-1,78$ & 0,1565 \\
$\geq 25$ & 54 & 18,43 & 86 & 29,66 & 0,54 & $0,36-0,81$ & 0,0016 \\
Total & 293 & 100,00 & 290 & 100,00 & & & \\
\hline
\end{tabular}

Fuente: Registro de Citologías Orgánicas. Policlínico Universitario “Dr. Juan Manuel Páez Inchausti”.

Tabla IV DISTRIBUCIÓN DE PACIENTES CON NEOPLASIA INTRAEPITELIAL (NIE) DE CUELLO UTERINO
SEGÚN TIEMPO DE USO DE ANTICONCEPTIVOS HORMONALES

\begin{tabular}{|c|c|c|c|c|c|c|c|}
\hline \multirow[t]{2}{*}{ Uso de $\mathrm{ACH}$} & \multicolumn{2}{|c|}{ Casos } & \multicolumn{2}{|c|}{ Controles } & \multirow[t]{2}{*}{ OR } & \multirow[t]{2}{*}{ IC 95\% } & \multirow[t]{2}{*}{ Valor $p$} \\
\hline & NIE & $\%$ & NIE & $\%$ & & & \\
\hline no uso & 239 & 75,63 & 246 & 77,85 & 0,88 & $0,60-1,30$ & 0,5102 \\
\hline$<5$ años & 43 & 13,61 & 47 & 14,87 & 0,90 & $0,56-1,44$ & 0,6492 \\
\hline $5-10$ & 21 & 6,65 & 19 & 6,01 & 1,11 & $0,56-2,21$ & 0,7441 \\
\hline > 10 años & 13 & 4,11 & 4 & 1,27 & 3,35 & $1,00-12,29$ & 0,0270 \\
\hline Total & 316 & 100,00 & 316 & 100,00 & & & \\
\hline
\end{tabular}

Fuente: Registro de Citologías Orgánicas. Policlínico Universitario “Dr. Juan Manuel Páez Inchausti”. $\mathrm{ACH}$ : anticonceptivos hormonales. 
Un análisis de los datos sobre mortalidad por cáncer cérvico uterino notificados por los países de América Latina y el Caribe indica que las mujeres entre los 35 y 54 años de edad conforman reiteradamente la mayor proporción de las defunciones anuales (19) En el año 2005, un tamizaje realizado en Perú bajo la supervisión de la OPS mostró un mayor riesgo de desarrollar la enfermedad en aquellas con edades comprendidas entre 35 y 50 años (20). Otros estudios muestran una mayor prevalencia a edades más precoces, como el realizado en Montevideo, Uruguay, en el 2003 donde se observó una concentración entre los 30 y 49 años con $67 \%$ (21). En Cuba se han reportado resultados similares. Un estudio realizado en Camaguey en el 2003 comprobó que el grupo etáreo más afectado es el que corresponde a las mujeres entre 35-59 años, hecho que se corresponde con la literatura revisada donde se considera esta enfermedad más frecuente en edades de mayor actividad sexual (22).

Estimamos que el haber encontrado la mayor prevalencia entre los 30-39 años está íntimamente relacionado con el inicio precoz de las relaciones sexuales que se observa en la población estudiada, asociado a la prevalencia - también alta - de infecciones cérvico-vaginales y en especial, de infección por el virus del papiloma viral humano.

Algunos estudios muestran predominio de la raza blanca en sus resultados como el realizado en el municipio de Cumanayagua, Cienfuegos, Cuba, con una prevalencia mayor al $90 \%$ (23). No se ha demostrado el mayor riesgo en diferentes razas o color de la piel; cuando se han encontrado diferencias, estas han sido relacionadas con factores socio-culturales y no raciales, por lo que se considera que los resultados encontrados en este estudio obedecen a la composición racial de la población estudiada (24).

Si bien no existe un planteamiento coincidente en el cáncer cérvico uterino en correspondencia con la menarquia precoz o tardía, como sucede por ejemplo con el cáncer de mama, si se plantea por algunos autores que han encontrado cierta correspondencia entre estas variables, como Benia y Tellechea en Uruguay (21), en cuyo estudio el 12,5\% de los casos tuvo una menarquia precoz. Otros estudios, como el de Castañeda y cols (26), que mostró asociación significativa cuando la menarquia ocurrió antes de los 10 años o después de los 14 años. Se considera que la edad de la menarquía por sí sola no representa un riesgo adicional, pero si se asocia con frecuencia al inicio de las relaciones sexuales, siendo interpretada por las adolescentes como expresión de madurez biológica; de manera que muchas adolescentes con menarquía precoz también inician precozmente su vida sexual.

Sin lugar a dudas, uno de los factores más importantes en la patogenia de la neoplasia cervical es el inicio precoz de la actividad sexual, relacionado este con una mayor probabilidad de asociación a infecciones cérvico vaginales y entre ellas, la infección por papiloma humano (HPV). Así, Benia y Tellechea (21), encontraron en Uruguay que uno de cada cinco casos de su estudio inició el sexo precozmente. El riesgo de neoplasia intraepitelial es mayor en la medida en que el inicio de la vida sexual activa es más temprano; así, en el estudio de Muñoz y cols (25), estimaron que aquellas que lo hicieron antes de los 15 años tienen un riesgo dos veces mayor (RM 2,0, IC95\% 1,2-3,5). Similares resultados encontró Torres-Lobaton (27), en Chihuahua (México), y Sun y cols (28) en su estudio en China.

Estimamos que el inicio precoz de la vida sexual, es esta una de las causas que ha motivado el desplazamiento de la incidencia de esta afección hacia edades menores. Con el inicio cada vez mas prematura de la vida sexual aumenta la probabilidad de que durante la metaplasia fisiológica de la adolescencia se perpetúe la infección por VPH y con ello se acelere la progresión natural de la enfermedad.

El VPH es una de las infecciones de transmisión sexual más comunes. Sin embargo, la infección desaparece o se torna indetectable en uno o dos años en la gran mayoría de los casos. Por ejemplo, entre una cohorte de mujeres universitarias sero-negativas al VPH, el 46 por ciento contrajo una infección por el VPH dentro de los tres años. Después de una mediana de seguimiento de 60 meses, la mayoría de estas infecciones por el VPH no progresaron a lesiones cervicales (29). Algunos estudios nacionales muestran resultados realmente significativos. Un estudio en Camaguey señaló que la infección por HPV estuvo presente en el $47,6 \%$ de los casos (22).

Los datos compartidos de ocho estudios de casos y controles sobre la NIC y el cáncer invasor del cuello uterino y dos estudios sobre el carcinoma in situ (CIS), efectuados en cuatro continentes, sugieren que las mujeres con tres o cuatro embarazos de término tenían un riesgo 2,6 veces más alto de aparición de cáncer cérvico uterino que las nulíparas; mientras que las mujeres con siete o más partos, presentaron un riesgo 3,8 veces mayor (30).

El motivo fisiológico de esta asociación es poco claro; las posibilidades incluyen factores hormonales vinculados al embarazo o traumatismo cervical asociado con el parto, el cual se hace más evidente cuando el primer parto ocurre precozmente y las 
condiciones cervicales no son óptimas para un parto transpelviano.

Investigaciones norteamericanas indican que hay una relación potencial a largo plazo entre el uso prolongado de anticonceptivos orales y la aparición de NIC y cáncer cérvico uterino. Un análisis de los datos compartidos de diez estudios de casos y controles sugiere que el uso a largo plazo de anticonceptivos orales podría aumentar hasta cuatro veces el riesgo de cáncer cérvico uterino en las mujeres infectadas con el VPH (31). Estimamos que aún faltan evidencias para demostrar esta asociación (32).

\section{CONCLUSIONES}

La neoplasia intraepitelial de cuello uterino fue más frecuente en nuestro estudio en las mujeres entre 35-39 años y en las de piel negra. Dentro de los factores de riesgo encontrados, resaltan el comienzo de las relaciones sexuales antes de los 15 años, la presencia del virus del papiloma humano, la paridad mayor o igual a cuatro y el uso de anticonceptivos hormonales por más de diez años.

\section{BIBLIOGRAFÍA}

1. Cabezas Cruz E. Conducta a seguir ante la NIC. Rev Cubana Obstet Ginec 1998;24(3):156-60.

2. De Palo G, Chaen W, Dexeus S. Neoplasia intraepitelial cervical. En: Patologia y tratamiento del tracto genital inferior. Barcelona: Editorial Masson; 2001.

3. Monnier-Benoit S, Dalstein V, Riethmuller D, Lalaoui $\mathrm{N}$, Mougin C, Prétet J. Dynamics of HPV16 DNA load reflect the natural history of cervical HPV-associated lesions. J Clin Virol 2006;35(3):270-7.

4. Fajardo Tornés Y, Lau Serrano D, Pérez Ramírez F. Experiencia en el tratamiento de la neoplasia intraepitelial cervical y el carcinoma in situ con radiocirugía. Rev Cubana Obstet Ginec 2010;36(2):112-20.

5. González M, Murillo R, Osorio E, Gamboa O, Ardila J. Prevalencia de anormalidades citológicas e histológicas de cuello uterino en un grupo de mujeres en Bogotá, Colombia. Rev Colomb Cancerol 2010;14(1):22-8.

6. International Agency for research on Cancer. Neoplasia maligna de cuello uterino. Tasas anuales promedio de mortalidad especifica por edad por 100000 personas en la region de las Américas. Globocan 2000, IARC.

7. Ministerio de Salud Publica. Programa Nacional de diagnóstico precoz del cáncer cervico uterino. Colectivo de autores. La Habana, 2001.

8. Cáncer cervico uterino: Dirección Nacional de Estadísticas. MINSAP, 2002.

9. Ministerio de Salud Pública. Anuario Estadístico 2006. La Habana [Citado 23 abril 2008]. Disponible en: http://www.bvs.sld.cu/cgi-bin/wxis/anuario

10. Bethesda workshop. The revised Bethesda system for reporting cervical and vaginal cytologic diagnoses. J Reprod Med 2001;37:383-6.
11. National Cancer Institute Workshop. The 2001 Bethesda system for reporting cervical and vaginal cytologic diagnoses. J Reprod Med 2002;34:779-85.

12. Cabezas E. Edad al inicio de las relaciones sexuales y carcinoma de cuello uterino. Rev Cubana Obstet Ginecol 2005;11(1):97-104.

13. Bosch FX, Lorincz A, Muñoz NC, Meijer JLM, Shah KV. The causal relation between human papillomavirus and cervical cancer. J Clin Pathol 2002;55(1):244-65.

14. Jastreboff AM, Cymet T. Role of the human papillomavirus in the development of cervical intraepithelial neoplasia and malignancy. Postgrad Med $\mathrm{J}$ 2002;78(1):225-8

15. Marielle AE. Relation of HPV status to cervical lesions and consequences for cervical cancer screening: a prospective study. Lancet 1999;354(9172):20-5.

16. Agorastos T, Miliaras D, Lambropoulos A, Chrisafi S, Kotsis A, Manthos A, Bontis J. Detection and typing of human papillomavirus DNA in uterine cervices with coexistent grade I and grade III intraepithelial neoplasia: biologic progression or independent lesions? Eur J Obstet Gynecol Reprod Biol 2005;121(1): 99-103.

17. Hillemanns $P$, Wang X, Staehle S, Michels W, Dannecker $\mathrm{C}$. Evaluation of different treatment modalities for vulvar intraepithelial neoplasia (VIN): CO2 laser vaporization, photodynamic therapy, excision and vulvectomy. Gynecol Oncol 2006;100 (2): 271-5.

18. Departamento de Estadísticas. Dirección Municipal de Salud Pública. Municipio Especial Isla de la Juventud. Registro de Defunciones 2007.

19. Barnum H, Greenberg ER. Cancers. In: Disease Control Priorities in Developing Countries. Eds: Jamison DT, Mosely WH, Measham AR and Bobadilla JL. Oxford University Press, 2003.

20. Organización Panamericana de la Salud. Prevención del cáncer cérvico-uterino en el Perú. Lecciones aprendidas del proyecto demostrativo TATI. 2006.

21. Benia W, Tellechea G. Estudio de los factores de riesgo para cáncer de mama y cuello uterino en mujeres usuarias de tres policlínicas barriales de Montevideo. Rev Med Uruguay 2004;16:103-13.

22. Amaro Hernández F, Polo Cardoso K. Comportamiento de algunos factores de riesgos asociados a la aparición del cáncer cérvico uterino en un área de Salud. Archivo Médico de Camagüey 2003;7(Supl. 2).

23. Bravo Polanco E, Rubio Rojas R, Co Marichal A. Factores de riesgo asociados al cáncer cérvico-uterino. Cumanayagua. Enero-Diciembre 2000. Disponible: http://www.aps.sld.cu/seminario2002/recursos/ver. php./factoresasociadosalcáncerdecuello.htm

24. Morales O, Pinedo T, Paricna J, Rubinos del Pozo J. Algunos aspectos epidemiológicos y clínicos de la infección por el PVH del cuello uterino: confirmación histológica. Acta Cancerol 2006;26(1):3-6.

25. Muñoz N, Bosch FX, De Sanjosé S, Viladiu P, Tormo J, Moreo $\mathrm{P}$, et al. El virus del papiloma humano en la etiología del cáncer cérvico uterino. PAHO Bull 1993;115(4):301-9.

26. Castañeda-Iñiguez MS, Toledo-Cisneros R, AguileraDelgadillo M. Factores de riesgo para cáncer cérvico uterino en mujeres de Zacatecas. Salud Pública Mex 2008;40:330-8. 
27. Torres-Lobaton A. Cáncer ginecológico diagnóstico y tratamiento. 2 ${ }^{\mathrm{a}}$ ed: México DF. Mc Graw Hill, 2003.

28. Sun CA, Liu JF, Wu DM, Nieh S, Yu CP, Chu TY. Viral load of high-risk human papillomavirus in cervical squamous intraepithelial lesions. Int J Gynaecol Obstet 2002;76:41-7.

29. Kjaer S, De Villiers E, Hangaard B, Chistensen R, Teisen C. Human papillomavirus, herpes simplex virus and cervical cancer incidence in Greenland and Denmark. A population-based cross sectional study. Int J Cancer 2004;41:518-24.
30. Bosch FX, De Sanjose S. Chapter 1. Human papillomavirus and cervical cancer-Burden and assessment of causality. J National Cancer Institute Monographs 2003;31:3-13.

31. Fasero Laíz M, Coronado Martín P, Costales Badillo C. Lesiones premalignas del cérvix y gestación. TokoGinecol Práctica 1999;58(3):133-8.

32. Becker MT, Wheeler C, McGough N, Stidley C, Parmenter $\mathrm{C}$, Dorin $\mathrm{M}$, et al. Contraceptive and reproductive risk for cervical dysplasia in Southwestern Hispanic and Non-Hispanic white women. Int $\mathrm{J}$ Epidemiol 2004;23(5):913-21. 